\title{
Lysophosphatidic Acid Regulates the Differentiation of Th2 Cells and Its Antagonist Suppresses Allergic Airway Inflammation
}

\author{
Mayo Kondo ${ }^{a}$ Toshifumi Tezuka ${ }^{a}$ Hirohisa Ogawab Kazuya Koyama ${ }^{a}$ \\ Hiroki Bando ${ }^{a}$ Masahiko Azuma ${ }^{a, c}$ Yasuhiko Nishioka ${ }^{a}$ \\ aDepartment of Respiratory Medicine and Rheumatology, Graduate School of Biomedical Sciences, Tokushima \\ University, Tokushima, Japan; 'D Department of Pathology and Laboratory Medicine, Graduate School of Biomedical \\ Sciences, Tokushima University, Tokushima, Japan; 'Department of Medical Education, Graduate School of \\ Biomedical Sciences, Tokushima University, Tokushima, Japan
}

\section{Keywords}

Lysophosphatidic acid · LPA2 antagonist · Allergic airway inflammation

\begin{abstract}
Background: Lysophosphatidic acid (LPA), a prototypic member of a large family of lysophospholipids, has been recently shown to play a role in immune responses to respiratory diseases. The involvement of LPA in allergic airway inflammation has been reported, but the mechanism remains unclear. Object: We analyzed the biological activity of LPA in vitro and in vivo and investigated its role in allergic inflammation in mice using an LPA receptor 2 (LPA2) antagonist. Methods: We used a murine model with acute allergic inflammation, in which mice are sensitized and challenged with house dust mite, and analyzed airway hyperresponsiveness (AHR), pathological findings, Th2 cytokines, and IL-33 in bronchoalveolar lavage fluid (BALF) and lung homogenates. The effect of LPA on Th2 differentiation and cytokine production was examined in vitro using naive $\mathrm{CD}^{+} \mathrm{T}$ cells isolated from splenocytes. We also investigated in vivo the effects of LPA on intranasal administration in mice. Results: The LPA2 antagonist suppressed the increase of AHR, the
\end{abstract}

karger@karger.com www.karger.com/iaa

Karger"

$$
\text { 兴 }
$$

number of total cells, and eosinophils in BALF and lung tissue. It also decreased the production of IL-13 in BALF and IL-33 and CCL2 in the lung. LPA promoted Th2 cell differentiation and IL-13 production by Th2 cells in vitro. Nasal administration of LPA significantly increased the number of total cells and IL-13 in BALF via regulating the production of IL-33 and CCL-2-derived infiltrating macrophages. Conclusion: These findings suggest that LPA plays an important role in allergic airway inflammation and that the blockade of LPA2 might have therapeutic potential for bronchial asthma.

(c) 2020 S. Karger AG, Basel

\section{Introduction}

Lysophosphatidic acid (LPA) is one of the lysophospholipids found mainly in blood plasma. LPA is derived from the hydrolysis of phosphatidylcholine, which is produced in liver by autotaxin (ATX). Lysophospholipids such as lysophosphatidylcholine and lysophosphatidylethanolamine, which are generated by activated platelets, are also a source of LPA [1-4]. LPA mediates a variety of

Edited by: H.-U. Simon, Bern.
(C) 2020 S. Karger AG, Base
Yasuhiko Nishioka

Department of Respiratory Medicine and Rheumatology

Graduate School of Biomedical Sciences, Tokushima University

3-18-15 Kuramoto-cho, Tokushima 770-8503 (Japan)

yasuhiko@tokushima-u.ac.jp 
biological responses, including cell proliferation, smooth muscle contraction, platelet aggregation, neurite retraction, and cell motility [1-3].

A previous report showed that LPA may be related to the pathogenic process of several respiratory inflammatory diseases. LPA levels were increased in bronchoalveolar lavage fluid (BALF) [5] and exhaled breath condensate of patients with idiopathic pulmonary fibrosis [6]. Moreover, previous reports have shown that LPA plays an important role in immune responses. It has been reported that LPA enhanced IL-13 gene expression in T cells in vitro [7]. LPA and eotaxin induced chemotaxis and the Ca flux response of Th2 cells [8]. LPA and ATX were implicated in lymphocyte trafficking and the regulation of lymphocyte entry into lymph nodes by producing high local concentrations of LPA in the high endothelial venules of lymph nodes $[9,10]$. Furthermore, LPA was shown to activate other immune cells such as neutrophils, mast cells, and macrophages [11]. In the airway structural cells, LPA stimulated IL-8 production in human bronchial epithelial cells by activating p38 MAP kinase and JNK [12]. LPA enhanced the contraction of airway smooth muscles [13]. These findings led to our hypothesis that LPA may be related to the exacerbation of allergic lung inflammation. Previous reports as described below support our hypothesis. LPA in BALF in bronchial asthma patients was significantly increased after allergen challenge $[1,14]$. In vivo studies with guinea pigs showed that inhalation of LPA increased the numbers of total cells, neutrophils, and eosinophils in the BALF [15].

There are 6 receptors LPA1-LPA6, which are highaffinity G-protein-coupled receptors [1-3]. Experimental mice administered an LPA1 antagonist [16] or mice deficient in LPA1 were found to have strong protection from lung fibrosis and mortality [5]. Similarly, LPA2 deficiency also protected against bleomycin-induced lung injury and fibrosis [17]. Some reports showed that LPA/LPA receptors were also related to pathogenesis in arterial sclerosis and rheumatoid arthritis $[18,19]$. The siRNAs of LPA1-3 were effective in reducing IL-8 production in bronchial epithelial cells in vitro [12]. In bronchial asthma, LPA2 $2^{+/-}$mice exposed to Schistosoma mansoni eggs had a significant reduction in Th2-dominant airway inflammatory responses [20]. Importantly, LPA1-4 are expressed on bronchial epithelium in the order of LPA2 > LPA $4>$ LPA1 $\geq$ LPA3 in mouse tracheal epithelial cells [20]. LPA2 mRNA is highly expressed in the lungs of mice [20]. According to these previous reports, LPA1-3 may have efficacy as a target molecule for controlling respiratory disease, and, in particular, LPA2 antagonists may be attractive agents for suppressing LPA receptor function in bronchial asthma. However, there are no reports describing the effects of LPA2 antagonists in bronchial asthma.

H2L5186303 ((Z,Z)-4,4'-[1,3-phenylenebis(oxy-4,1phenyleneimino)]bis[4-oxo-2-butenoic acid]) is a selective LPA2 receptor antagonist $\left(\mathrm{IC}_{50}=9 \mathrm{nM}\right)$, which does not inhibit LPA1 and LPA3 receptors, as they need much higher concentrations to inhibit them $\left(\mathrm{IC}_{50}=27,354\right.$ and 4,504 nM, respectively) (Cayman Chemicals, Dallas, TX, USA). H2L5186303 prevented intercellular gap formation in endothelial cells and suppressed atherosclerosis [4]. The ATX/LPA axis contributes to tumor cell progression including fibrosarcoma and breast cancer in vitro, and H2L5186303 inhibited tumor cell proliferation [21, 22].

In this report, we investigated if LPA contributes to allergic airway inflammation and showed that it promotes the differentiation of Th2 cells, leading to the induction of allergic inflammation. Moreover, we showed that an antagonist of LPA2 was effective in suppressing these responses.

\section{Materials and Methods}

Detailed methods are described in the online supplementary material (see www.karger.com/doi/10.1159/000509804 for all online suppl. material).

\section{Antigen and Agent Preparation}

House dust mite antigen (Dermatophagoides pteronyssinus, Dp) was purchased from Cosmo Bio (Tokyo, Japan). Endotoxin removal solution (Sigma-Aldrich Japan, Tokyo, Japan) was used to reduce the endotoxin concentration. After removal, Dp endotoxin levels were under $0.5 \mathrm{IU} / \mathrm{mg}$.

LPA was purchased from Santa Cruz Biotechnology (Dallas, TX, USA). It was dissolved in normal saline at the concentration described in each experiment. The potent and selective LPA2 receptor antagonist, H2L5186303 (Z,Z)-4,4' - [1,3-phenylenebis(oxy4,1-phenyleneimino)]bis[4-oxo-2-butenoic acid] was purchased from R\&D Systems, Inc. (Minneapolis, MN, USA). It was dissolved in DMSO to $100 \mathrm{mM}$. IL-33 was purchased from R\&D Systems, Inc.

\section{Mouse Experimental Protocols}

Five-week-old female BALB/c mice were purchased from CLEA Japan, Inc. (Tokyo, Japan). Mice were maintained in the animal facility of Tokushima University under specific pathogenfree conditions, according to the guidelines and approval of the ethics committee of our university [23].

We used 2 different models. In the first protocol, we generated acute allergic inflammation model with LPA2 antagonist treatment (Fig. 1a). On days 0 and 7, mice were sensitized by intraperitoneal injections of $10 \mu \mathrm{g}$ of Dp dissolved in $500 \mu \mathrm{L}$ saline and mixed with $1 \mathrm{mg}$ of alum (Sigma-Aldrich Japan, Tokyo, Japan), and all mice were then challenged intranasally with $10 \mu \mathrm{g}(10 \mu \mathrm{L})$ 


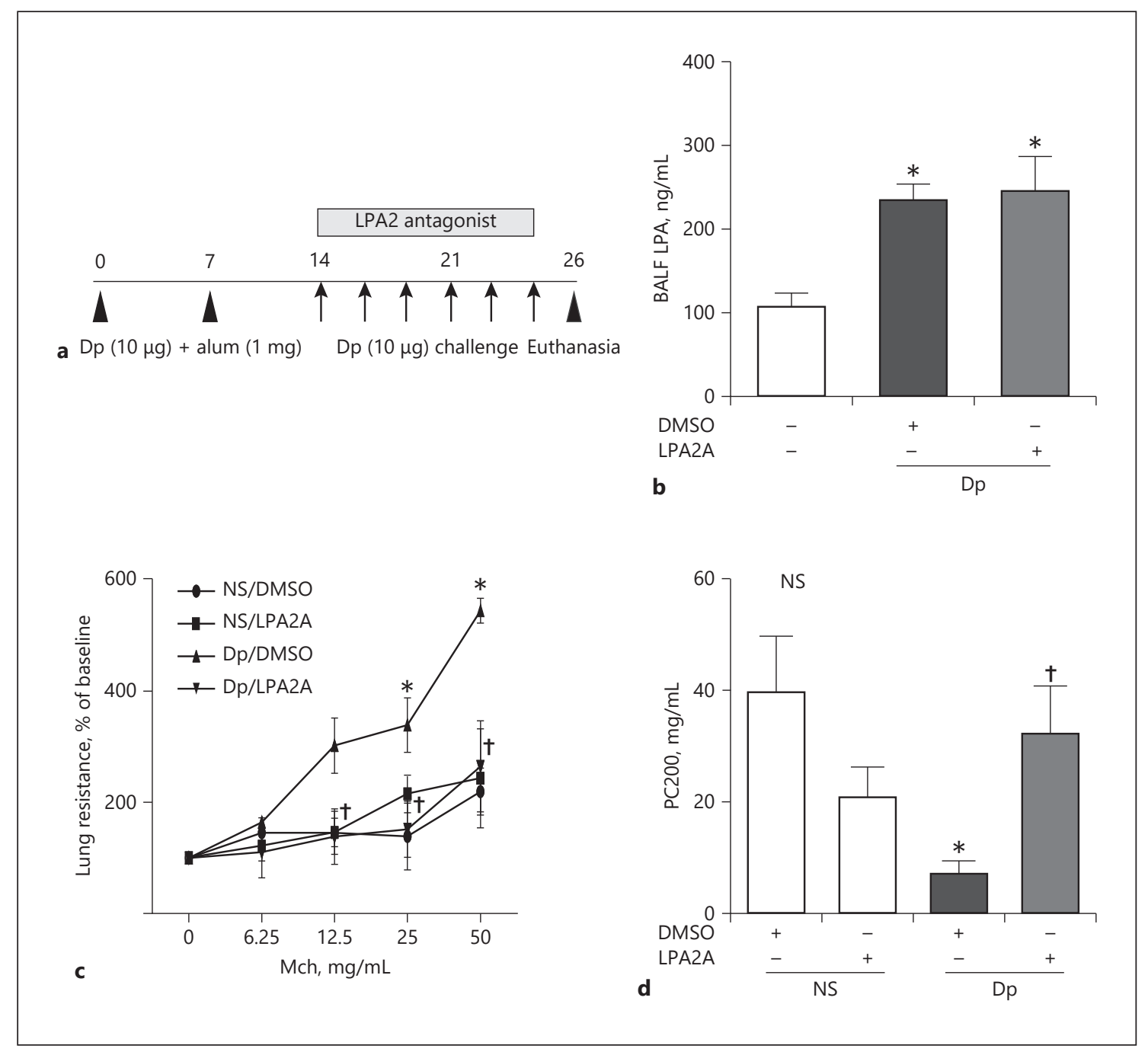

Fig. 1. Assessment of airway resistance and PC200 after Dp challenge. a Protocols for LPA2 antagonist treatment model. b The concentration of LPA in BALF in mouse model. LPA concentration was measured by ELISA. Data are expressed as means $\pm \mathrm{SE}$ and are representative of 2 independent experiments. White bar: control $(n=7)$. Black bar: Dp/DMSO $(n=8)$. Shaded bar: Dp/ LPA2 antagonist $(n=7)$. c Assessment of airway resistance after $\mathrm{Dp}$ challenge and LPA2 antagonist treatment. $\mathbf{d}$ The value of

of $\mathrm{Dp}$ in $60 \mu \mathrm{L}$ saline every other day, 3 days per week, from day 14 to 25 . LPA2 antagonist ( $10 \mu \mathrm{g} / \mathrm{mouse})$ was administered intranasally into Dp-challenged mice 30 min before Dp challenge. Mice were euthanized on day 26.

The second model was an LPA intranasal administration model, which investigated the inflammatory effects of LPA on bronchial allergic inflammation. All mice were challenged intranasally with 10,50 , and $100 \mu \mathrm{g}$ (in $10 \mu \mathrm{L}$ ) of LPA in $60 \mu \mathrm{L}$ saline on days $0,2,4,6$, and 8 . Mice were euthanized on day 9 (Fig. $5 \mathrm{a}$ ).
PC200 assessed by airway hypersensitivity following Dp challenge and LPA2 antagonist treatment. Data are expressed as means \pm SE of 6 mice and are representative of 3 independent experiments. ${ }^{*} p<0.05$ compared with NS/DMSO mice. ${ }^{\dagger} p<0.05$ compared with Dp/DMSO mice. Dp, Dermatophagoides pteronyssinus; LPA, lysophosphatidic acid; LPA2, LPA receptor 2; BALF, bronchoalveolar lavage fluid.
IL-33 was administered intranasally, using the same protocol as for the LPA intranasal administration model, as a positive control model in order to investigate the innate lymphoid cells (ILC) 2. All mice were challenged intranasally with $500 \mathrm{ng}$ of IL33 in $70 \mu \mathrm{L}$ saline on days $0,2,4,6$, and 8 , and were euthanized on day 9. Each experiment was performed using 5-6 mice per group. 
Plethysmography

Lung resistance $\left(R_{\mathrm{L}}\right)$ was measured by restrained whole-body plethysmography (Buxco Electronics, Troy, NY, USA). The provocative concentration of Mch that caused a $200 \%$ increase in $R_{\mathrm{L}}$, PC200, was calculated by linear interpolation of the dose-response curves [24].

\section{Histopathology and Immunohistochemistry}

The lung tissue was fixed in 10\% formalin and embedded in paraffin. Three-micrometer-thick sections were stained with Luna and periodic acid-Schiff (PAS). A BZ-9000 microscope (KEYENCE, Itasca, IL, USA) was used for images and ImageJ software was used for the morphological analysis.

Immunohistochemistry was performed using anti-mouse CD68 (Abcam, Cambridge, UK). An OLYMPUS BX61 microscope was used for images and Image J software (National Institute of Health) was used for morphological analysis. Hot spots of CD68-positive cells at 20 fields were counted and averaged out.

\section{Enzyme-Linked Immunosorbent Assays}

Cytokines, LPA, and LPA receptors were determined using commercial ELISA kits according to the manufacturer's instructions. ELISA kits and their sensitivities were as follows: IL-5, IL13, IL-33, and CCL2 (R\&D Systems, Inc., Minneapolis, MN, USA) with sensitivities of $7,1.5,14.3$, and $7.58 \mathrm{pg} / \mathrm{mL}$, respectively; LPA (Cloud-Clone Corp., Katy, TX, USA) with a sensitivity of $<52.7 \mathrm{ng} / \mathrm{mL}$; and LPA1 and LPA2 (MyBioSource, Inc., San Diego, CA, USA) with sensitivities of $<0.098$ and $0.1 \mathrm{ng} / \mathrm{mL}$, respectively.

\section{Isolation and Stimulation of Naive T Cell Harvest}

Spleens were harvested from mouse and were homogenized by passage through stainless steel mesh. Cells were suspended in Red Blood Cell Lysing Buffer, Hybri-Max ${ }^{\mathrm{TM}}$ (Sigma-Aldrich Japan, Tokyo, Japan). Next, naive CD4 ${ }^{+} \mathrm{T}$ cells were isolated from the cell suspensions using auto-MACS (Miltenyi Biotec K.K., Bergisch Gladbach, Germany) with Naive CD4 ${ }^{+}$T Cell Isolation Kits (Miltenyi Biotec K.K.) according to the manufacturer's instructions. Naive T cells were plated in 48 -well cell culture plates at a final concentration of $1 \times 10^{6}$ cells/well using TexMACS Medium with CytoBox, Th2 Cell Kit (Miltenyi Biotec K.K.). Then, T cells were incubated with beads coated with CD3/CD28 antibody (T Cell Activation Expansion kit; Miltenyi Biotec K.K), LPA, LPA1 antagonists, and LPA2 antagonists for $24 \mathrm{~h}$ to evaluate LPA and its antagonist effect on Th2 differentiation. T cells were collected after LPA, LPA1 antagonist, and LPA2 antagonist stimulation and were used in flow cytometry studies. Supernatants were also harvested and stored at $-80^{\circ} \mathrm{C}$ for the measurement of cytokines.

Isolation and Analysis of ILC2s

ILC2s were collected from 5 murine spleens and 5 murine pairs of lungs. Lungs were minced and digested with DNase I (Roche, Branford, CT, USA) and collagenase I (Roche) for $1 \mathrm{~h}$ at $37^{\circ} \mathrm{C}$ to obtain single cell suspensions. Spleens were minced to obtain splenocytes. After mashing through $100-\mu \mathrm{m}$ cell strainers, the cells were incubated with anti-CD16/CD32 to block Fc receptors before antibody staining. The cells were stained with antibodies for lineage markers (CD3, CD45R, CD11b, TER-119, and Gr-1), CD127, CD25, CD117/c-kit, and Sca-1. ILC2s were identified as $\mathrm{Lin}^{-} \mathrm{Sca}-$ $1^{+} \mathrm{C}-\mathrm{Kit}^{+} \mathrm{CD} 127^{+} \mathrm{CD} 25^{+}$cells and isolated on a Sony LE-SH800 cell sorter (Sony Biotechnology Inc., San Jose, CA, USA) with a 100$\mu \mathrm{m}$ sorting chip.

The isolated ILC2s were cultured at a concentration of 2,500 cells per well in 96-well round bottom plates using base medium. In order to evaluate the production of IL-13, the cells were incubated with IL-2 $(20 \mathrm{ng} / \mathrm{mL})$ and IL-7 $(20 \mathrm{ng} / \mathrm{mL})$ and stimulated with LPA (1 nM), IL-33 (100 ng/mL), and LPA (1 nM) + IL-33 (100 $\mathrm{ng} / \mathrm{mL}$ ) for 4 days. The supernatant was harvested and stored at $-80^{\circ} \mathrm{C}$.

\section{Flow Cytometric Analysis}

T cells and ILC2 cells were incubated with anti-CD16/CD32 to block Fc receptors before staining and were stained with the antibodies described online suppl. material. Th2 cells were sorted and analyzed on an LE-SH800BC cell sorter (Sony Japan, Tokyo, Japan; online suppl. Fig. 1). The numbers of ILC2 were analyzed with BD LSRFortessa (BD Biosciences, San Diego, CA, USA).

\section{Statistical Analysis}

Experimental results were expressed as mean \pm SE. Experimental groups were compared using 1-way ANOVA. If statistical significance was identified by ANOVA, a Tukey post hoc test was used to correct for multiple comparisons. Statistical analyses were performed using GraphPad PRISM software (5.01; GraphPad Software, Inc., LA Jolla, CA, USA). $p$ values $<0.05$ were considered significant.

\section{Results}

\section{LPA Increase in Allergic Inflammation and \\ Inhibition of Airway Hyperresponsiveness by an \\ LPA2 Antagonist}

First, we examined if LPA is elevated in the lungs of our allergic inflammation model. The value of LPA in the BALF of Dp/DMSO was significantly increased compared to control/DMSO mice (Fig. 1b). LPA2 antagonist H2L5186303 treatment did not affect the LPA level in the BALF (Fig. 1b). To examine the effects of H2L5186303 on airway hyperresponsiveness (AHR) in the model, we assessed lung resistance $\left(R_{\mathrm{L}}\right)$ and AHR using PC200 by restrained plethysmography. Repeated-measures 2-way ANOVA showed that the curves for all groups were significantly different. The value of $R_{\mathrm{L}}$ over $25 \mathrm{mg} / \mathrm{mL}$ of methacholine in $\mathrm{Dp} /$ DMSO mice was greater than in NS/DMSO mice. H2L5186303 significantly reduced airway resistance in Dp/ LPA2A mice compared to that in Dp/DMSO mice over $12.5 \mathrm{mg} / \mathrm{mL}$ of Mch (Fig. 1c). Regarding PC200, the AHR of Dp/DMSO mice was significantly decreased compared to that of NS/DMSO mice (Fig. 1d). However, the AHR of Dp/LPA2A mice was significantly restored compared to that of Dp/DMSO mice (Fig. 1d). These findings suggest that administration of an LPA2 antagonist improved AHR in an allergic inflammation mouse model. 


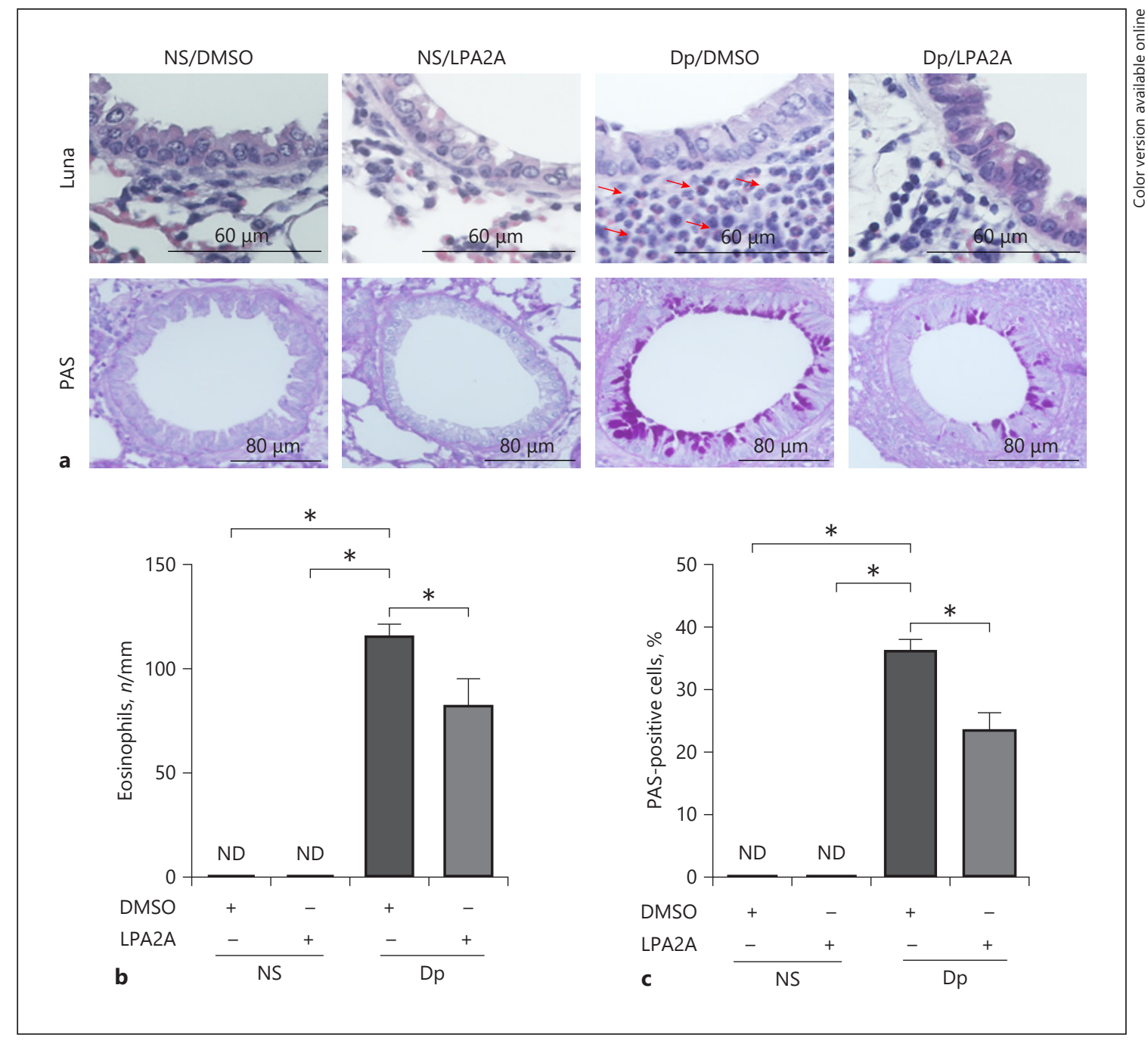

Fig. 2. Histopathology of allergic inflammation. a Histopathology in the lungs. Upper panel: Luna stains (original magnification in all figures, $\times 400$. Scale bars in all figures, $60 \mu \mathrm{m})$. Lower panels: PAS stains (original magnification in all figures, $\times 200$. Scale bars in all figures, $80 \mu \mathrm{m})$. $\mathbf{b}$ The number of eosinophils in the subepithelium. c The percentage of PAS-positive cells. Data are expressed as means \pm SE of 6 mice and are representative of 3 independent experiments. ${ }^{*} p<0.05$. ND, not detected. Black bars: Dp/DMSO $(n=6)$. Shaded bars: Dp/LPA2 antagonist $(n=6)$. PAS, periodic acid-Schiff; Dp, Dermatophagoides pteronyssinus; LPA2, lysophosphatidic acid receptor 2 .

\section{Inhibition of Allergic Inflammation by an LPA2 Antagonist}

In the LUNA-stained lung sections, eosinophils had infiltrated in Dp/DMSO mice following Dp challenge, although they were decreased when the mice were treated with H2L5186303 (Dp/LPA2A) (Fig. 2a). The number of infiltrating eosinophils under the subepithelium increased in Dp/DMSO mice compared to NS/DMSO mice (Fig. 2b). Treatment with H2L5186303 significantly decreased the numbers of infiltrating eosinophils (Fig. 2b). The PAS staining also showed mucous metaplasia in air- way epithelial cells in Dp/DMSO mice (Fig. 2a), and the PAS-positive cells were decreased by treatment with H2L5186303 (Fig. 2a). The percentage of PAS-positive area in Dp/DMSO mice was increased compared to that in Dp/LPA2A mice (Fig. 2c).

\section{Inhibition of Inflammatory Cell Infiltration and \\ Cytokine Production by an LPA2 Antagonist}

We next assessed airway inflammation using BALF. BALF was collected and cell classifications were performed as described in the Materials and Methods. The 

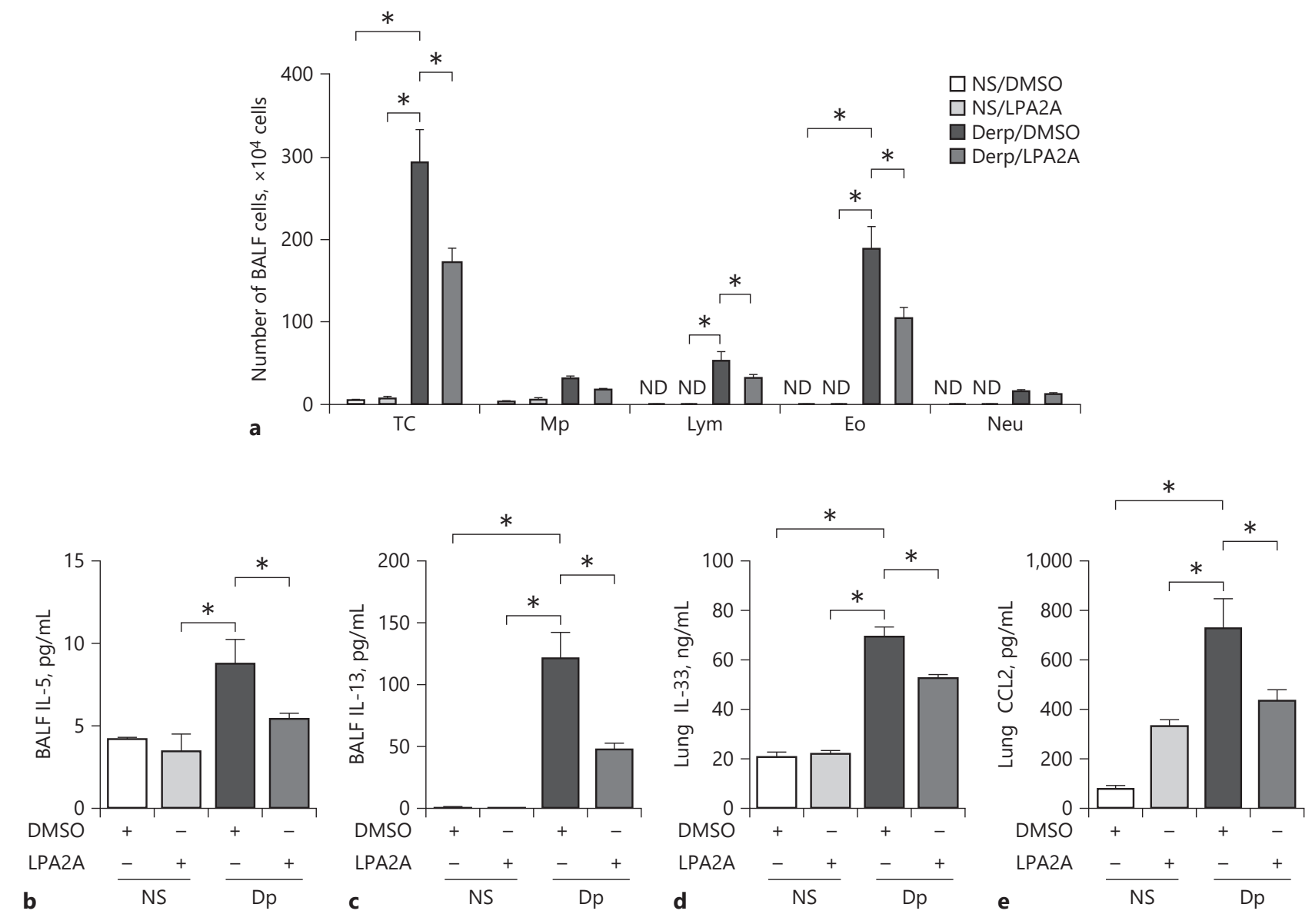

Fig. 3. Analyses of cell classification and cytokines in BALF. a BALF cell analysis. Cytokine levels in mouse BALF (b, c) and lung homogenates (d, e). IL-5 (b), IL-13 (c), IL-33 (d), and CCL2 (e) were measured by ELISA. Data are expressed as means \pm SE of 6 mice, and are representative of 3 independent experiments. ${ }^{*} p<$ 0.05 . White bars: control $(n=6)$ and dotted bars: NS/LPA2 an-

total cell counts in the BALF in Dp/DMSO mice were significantly higher than those in Dp/LPA2A mice (Fig. 3a). In particular, eosinophils increased in Dp/DMSO mice compared to those in NS/DMSO mice (Fig. 3a). On the other hand, treatment with H2L5186303 significantly decreased the numbers of eosinophils in Dp/LPA2A mice, as compared with Dp/DMSO mice (Fig. 3a).

The levels of Th2 cytokines, including IL-5 and IL-13, in Dp/DMSO mice were higher than those in control/ DMSO mice; administration of H2L5186303 significantly decreased the production of these interleukins (Fig. 3b, c). We also examined IL-33 in lung homogenates, as it was not detected in the BALF. The value of IL-33 in the tagonist $(n=6)$. Black bars: Dp/DMSO $(n=6)$. Shaded bars: Dp/ LPA2 antagonist $(n=6)$. BALF, bronchoalveolar lavage fluid; TC, total cells; Mp, macrophage; Lym, lymphocytes; Eo, eosinophils; Neu, neutrophils; Dp, Dermatophagoides pteronyssinus; LPA2, lysophosphatidic acid receptor 2 .

lung of Dp/DMSO was significantly increased compared to that of NS/DMSO mice (Fig. 3d). Treatment with H2L5186303 significantly decreased IL-33 production in Dp/LPA2A mice (Fig. 3d). The value of CCL2 in the lungs of Dp/DMSO was significantly increased compared to that of NS/DMSO mice, and administration of H2L5186303 significantly decreased CCL2 production (Fig. 3e).

\section{Effect of LPA on the Differentiation of Naive T Cells} into Th2 Cells

Previous reports demonstrated that blocking ATX activity or knockdown of the LPA2 receptor in mice pro- 


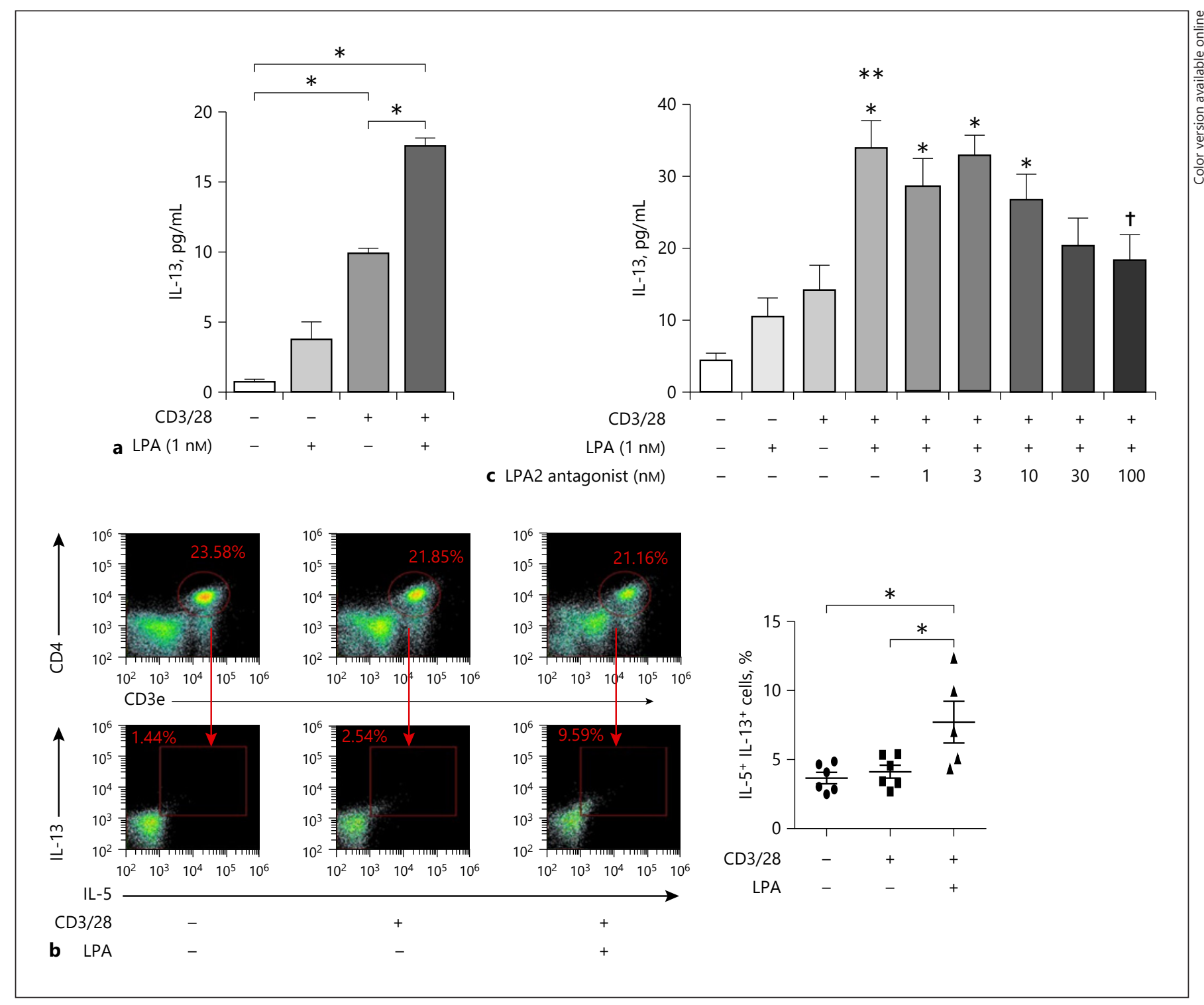

Fig. 4. Effect of LPA on Th2 differentiation of naive T cells. a Cytokine IL-13 levels in the supernatants of T cells after CD3/28 (T cell activation) and LPA stimulation. IL-13 was measured by ELISA. White bar: control $(n=6)$. Black bar: LPA stimulation $(n=6)$. Dotted bar: CD3/28 stimulation $(n=6)$. Shaded bar: LPA and CD3/28 stimulation $(n=6)$. $\mathbf{b}$ Left panel shows representative flow cytometry analysis of the IL- $5^{+} \mathrm{IL}-13^{+} \mathrm{T}$ cells. Right panel revealed the $\%$ of IL- $5^{+}$IL- $13^{+}$cells. c The effect of LPA2 antagonist on IL13 levels in the supernatants of T cells after CD3/28 and LPA stim-

duced a marked attenuation of Th2 cytokines and allergic lung inflammation [1]. Thus, we examined the expression of LPA receptors on naive T cells, and LPA2 receptors were more highly expressed on T cells compared to expression of LPA1 (LPA1 vs. LPA2 $=2.1 \pm 0.17$ vs. $3.3 \pm$ 0.33 , respectively; online suppl. Table 1 ). Therefore, we

Lysophosphatidic Acid Induces Allergic Inflammation ulation. White bars: control $(n=6)$. Black bars: LPA stimulation with or without CD3/28 $(n=6)$. Dotted bars: CD3/28, Shaded bar: CD3/28 and LPA plus LPA2 treatment $(n=6)$. These data are expressed as means \pm SE of 6 mice and are representative of 2 independent experiments. ${ }^{*} p<0.05$ compared to control, ${ }^{* *} p<0.05$ compared to $\mathrm{CD} 3 / 28$ stimulation. ${ }^{\dagger} p<0.05$ compared to LPA + CD3/28 stimulation. LPA, lysophosphatidic acid; LPA2, LPA receptor 2 .

hypothesized that the LPA/LPA2 axis may have a synergistic effect on Th2 differentiation. To clarify this, under Th2-inducible conditions, we assessed $\mathrm{T}$ cell differentiation by stimulation with LPA in vitro and assessed the inhibitory effect of the LPA2 antagonist. As shown in Figure $4 \mathrm{a}, \mathrm{IL}-13$ production in T cells stimulated by $1 \mathrm{nM}$ of 


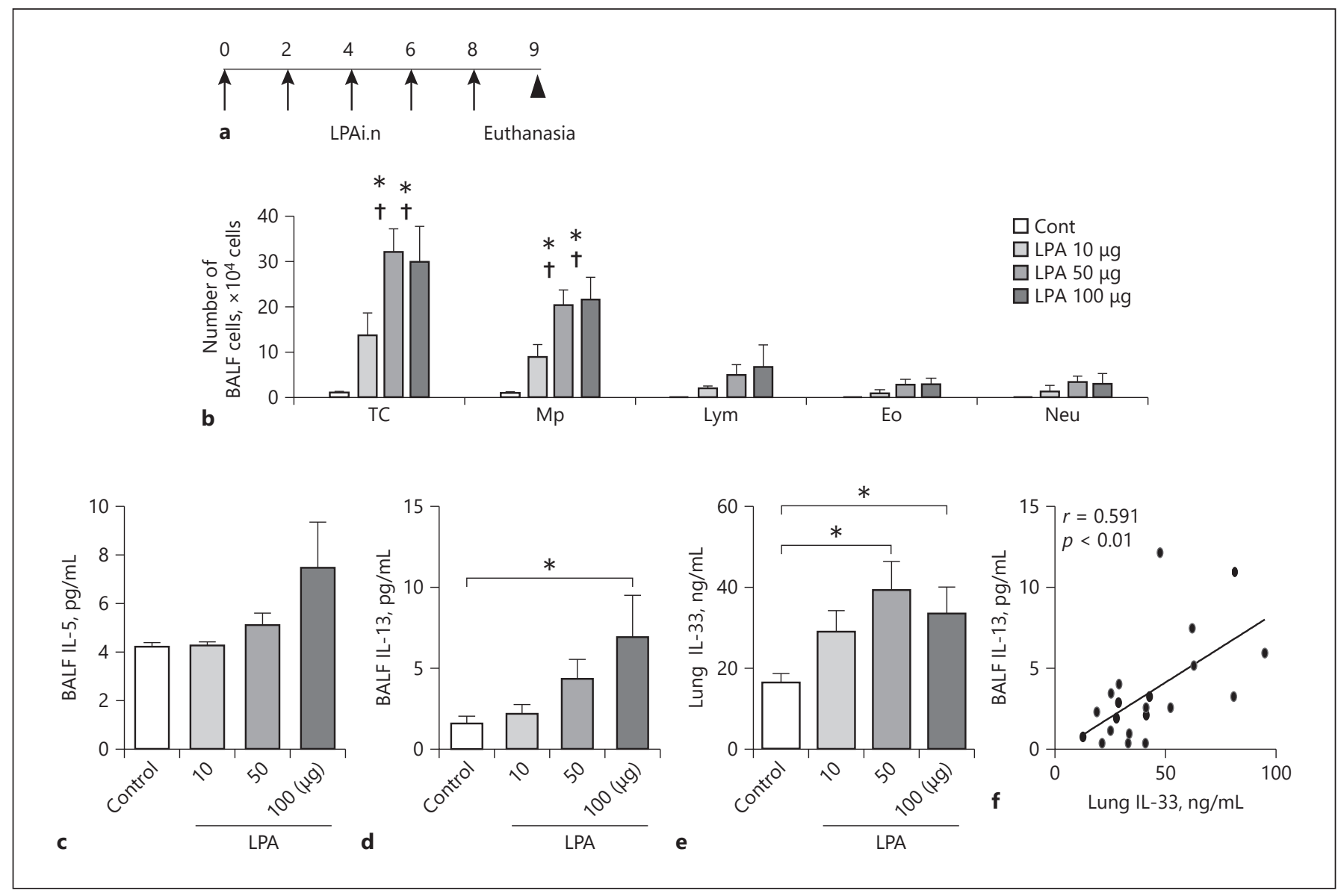

Fig. 5. IL-13 and IL-33 production following LPA intranasal administration. a Protocol. b BALF cell analysis. Cytokine levels in mouse BALF IL-5 (c), IL-13 (d), and lung homogenates IL-33 (e). Cytokines were measured by ELISA. Data are expressed as means \pm SE of 6 mice and are representative of 2 independent experiments. ${ }^{*} p<0.05$ compared with control mice. ${ }^{\dagger} p<0.05$ compared $10 \mu \mathrm{g}$ of LPA treatment. White bars: control $(n=6)$. Black bars: LPA treatment group $(n=6)$. $f$ Correlation between lung homogenate IL-33 and BALF IL-13. $x$ axis: lung IL-33. $y$ axis: BALF IL-13. TC, total cells; Mp, macrophage; Lym, lymphocytes; Eo, eosinophils; Neu, neutrophils; LPA, lysophosphatidic acid; BALF, bronchoalveolar lavage fluid.
LPA tended to increase compared to unstimulated T cells. Although $\mathrm{T}$ cell activation using beads coated with CD3/ CD28 antibodies (CD3/28) increased IL-13 production in $\mathrm{T}$ cells, IL-13 production was significantly increased in $\mathrm{T}$ cells co-stimulated with anti-CD3/28 and $1 \mathrm{nM}$ of LPA compared to those stimulated with anti-CD3/28 alone (Fig. 4a). By flow cytometry, the percentage of IL- $5^{+} \mathrm{IL}-13^{+}$ T cells (Th2 cells) incubated with anti-CD3/28 and $1 \mathrm{~nm}$ of LPA was increased compared to T cells incubated in both medium and with anti-CD3/28 (Fig. 4b). The LPA2 antagonist H2L5186303 significantly decreased IL-13 production in a dose-dependent manner (Fig. 4c). On the other hand, the LPA1 antagonist, AM095, did not affect IL-13 production (online suppl. Fig. 3). These findings suggest that LPA may have a synergistic effect on the dif- ferentiation of naive $\mathrm{T}$ cells into $\mathrm{Th} 2$ cells. In addition, the main functional receptor impacting the differentiation of Th2 cells may be LPA2.

\section{LPA Induces IL-33 Production}

We showed that abundant IL-33 production in Dp/ DMSO mice was decreased by LPA2 antagonist treatment (Fig. 3d), leading us to consider that LPA contributes to IL-33 production directly. To clarify this, we examined the BAL cytology and histopathology of the experimental group administered LPA (Fig. 5a). As shown in Figure 5b, the numbers of total cells and macrophages were increased in an LPA dose-dependent fashion $(10,50$, and $100 \mu \mathrm{g})$ compared to the control group. LPA administration induced a significant increase in 


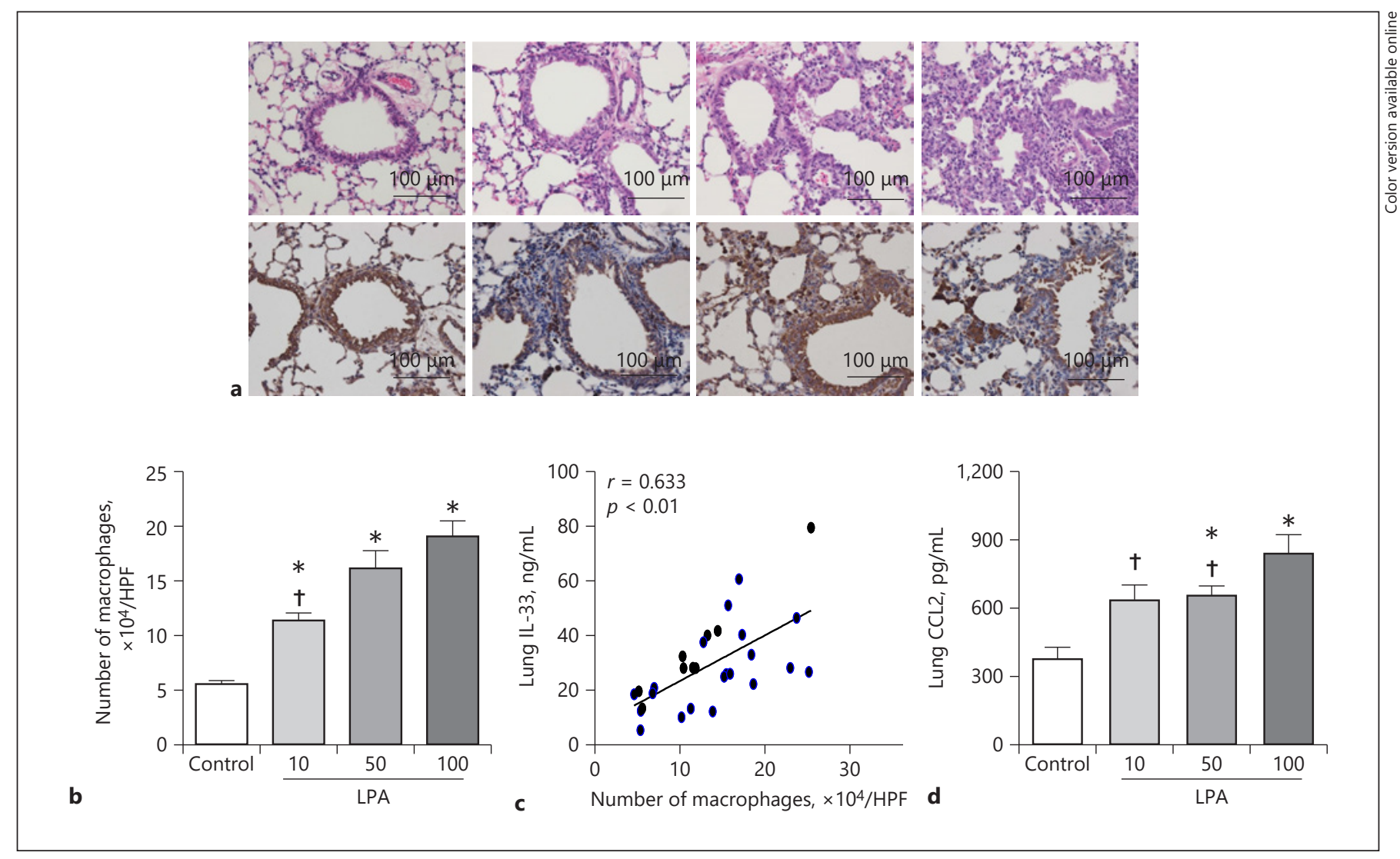

Fig. 6. Macrophage infiltration and IL-33 production in LPA intranasal administration. a Histopathology (HE and CD68) in the lungs. Original magnification in all figures, $\times 100$. Scale bars in all figures, $100 \mu \mathrm{m}$. b The number of CD68 positive cells (macrophages). Data are expressed as means \pm SE of 6 mice and are representative of 3 independent experiments. ${ }^{*} p<0.05$ compared with control group. ${ }^{\dagger} p<0.05$ compared $100 \mu \mathrm{g}$ of LPA treatment. White bars: control $(n=6)$. Black bar: LPA treatment group. c Correla- tion between macrophages and lung homogenate IL-33. $x$ axis: macrophage (CD68 ${ }^{+}$cells). $y$ axis: lung IL-33. d Levels of CCL2 in mouse lung homogenates. Cytokines were measured by ELISA. Data are expressed as means \pm SE of 6 mice and are representative of 2 independent experiments. ${ }^{*} p<0.05$ compared with control mice. ${ }^{\dagger} p<0.05$ compared $100 \mu \mathrm{g}$ of LPA treatment. LPA, lysophosphatidic acid. the levels of IL-13 in the BALF in a dose-dependent manner compared with those of control mice, but did not affect IL-5 production (Fig. 5c, d). As shown in Figure $5 \mathrm{e}$, nasal administration of LPA significantly increased IL-33 in lung homogenate (Fig. 5e). Similarly, IL-33 in lung homogenate correlated with IL-13 in $\operatorname{BALF}(R=0.5910$ and $p=0.0048)$, indicating that IL-33 increased by LPA stimulated the production of IL- 13 in BALF (Fig. 5f). These findings indicated that LPA also contributed to the induction of bronchial allergic inflammation through IL-33 production as well as Th2 differentiation.

\section{LPA Contributes to IL-33 Production by Limiting the} Infiltration of Macrophages

IL-33 was produced by bronchial epithelial cells, mast cells, and macrophages by various stimuli [25]. Therefore, we next examined the cellular sources of IL-33 production in mice that received intranasally administered LPA. Initially, we examined bronchial epithelial cells, but the production of IL-33 was not detected in bronchial cells stimulated with LPA (data not shown). Therefore, we focused on macrophages as the source of IL-33. CD68positive cells (macrophages) infiltrated following LPA stimulation (Fig. 6a). The number of CD68-positive cells in the LPA groups was increased in a dose-dependent manner (Fig. 6b). In addition, the number of macro- 
phages showed a correlation with IL-33 in lung homogenate ( $r=0.6326$ and $p=0.0002)$, indicating that macrophages may play a role in the increase of IL-33 in lungs stimulated with LPA (Fig. 6c). In addition, we examined the influence of macrophage infiltration to the site of allergic inflammation through LPA2. As shown in online suppl. Table 1, LPA receptors were detected in BAL cells, but the levels of LPA1 were higher than those of LPA2 in BAL cells. CCL2 production increased in an LPA dosedependent manner (Fig. 6d), and administration of an LPA2 antagonist affected CCL2 production in lungs with allergic inflammation (Fig. 3e). These findings suggest that LPA induced CCL2 production, which resulted in macrophage infiltration and the IL-33 production. Moreover, these findings also suggest that the LPA2 antagonist suppressed the CCL2-derived macrophage infiltration.

\section{LPA Does Not Affect ILC2 Infiltration and Activation}

As shown in online suppl. Figure $2 \mathrm{a}$ and $\mathrm{b}$, the number of ILC2 was increased in acute allergic inflammation model, and it was suppressed by LPA2 antagonist. ILC2 were increased significantly by IL-33, not by LPA in nasal administration model (online suppl. Fig. $2 \mathrm{a}, \mathrm{b}$ ). In in vitro experiments, the value of IL-13 was significantly increased in the supernatants of ILC2 stimulated by IL-33, but it was not increased following LPA stimulation (online suppl. Fig. 2c).

\section{Discussion}

In the present study, we demonstrated that LPA played a role in allergic inflammation via differentiating Th2 cells and activating macrophages. In addition, LPA2 receptor blockade can have a potential therapeutic effect on bronchial asthma, and we hypothesized that inhibition of the LPA receptor by a receptor antagonist could control the exacerbation of asthma. We confirmed that LPA2 antagonist administration suppressed allergic inflammation, including the total number of cells, eosinophil infiltration, and increased Th2 cytokines in BALF in our asthma model. This suggests that LPA2 antagonists are effective therapeutic modalities in our acute allergic inflammation model.

Our data clearly show that LPA promoted Th2 differentiation synergistically with Th2 cytokines in vitro, probably through the LPA2 receptor. Previous reports showed that LPA and the related lysophospholipids had various synergistic effects, including apoptosis, cell migration of T cells, and cytokine synthesis [26-28]. LPA1-
3 receptors are expressed in T cells $[7,8]$. Rubenfeld et al. [7] reported that LPA stimulation increased IL-13 production following CD3 and CD28 stimulation of CD $4^{+} \mathrm{T}$ cells harvested from human peripheral blood and that LPA activated the IL-13 promoter via regulatory elements contained within the proximal $312 \mathrm{bp}$, which were distinct from GATA-3. In the present findings, we also demonstrated a similar result for $\mathrm{CD} 4^{+} \mathrm{T}$ cells harvested from mouse spleen by flow cytometry. Moreover, we showed that the increase in IL-13 production by LPA stimulation was inhibited by LPA 2 antagonist treatment in an allergic inflammation model. These findings suggest that the LPA/LPA2 axis may be important for the Th2-driven inflammation seen in acute allergic inflammation.

Recently, regarding Th2 skewing, it is well known that IL-33 induces the ILC2 to produce Th2 cytokines, including IL-5 and IL-13 [29, 30]. Recent studies have shown that Th2-high late-onset asthma was severe and less effected by inhaled corticosteroids, indicating that the main causal factor of this phenotype was not Th2 cells but the innate lymphoid response, including ILC2 [31]. Other studies have reported that lipid mediators such as LTD4 and PGD2 activated ILC2 directly and not via IL-33 and IL-25 $[32,33]$. These findings suggest that LPA may contribute to the refractory nature of asthma, as our data also showed that an LPA2 antagonist controlled IL-33 production. Hence, with reference to the previous report and our findings, we considered the possibility that LPA activates ILC2 directly and investigated the effect of LPA2 on ILC2, as described above. In in vivo experiments, the number of ILC2 was increased in the acute allergic model we employed, and their number was markedly increased by IL-33 nasal administration. However, it increased very little following LPA nasal administration. Moreover, in in vitro experiment, the level of the Th2 cytokine IL-13 was significantly increased by IL-33 stimulation, but it was not significantly increased by LPA stimulation. These results suggest that LPA does not affect ILC2 directly but exacerbates allergic bronchial inflammation by promoting Th2 differentiation and IL-33 production.

Secondly, we demonstrated that the synergistic Th2 responses due to IL-33 stimulation were involved in LPA, probably through the activation of macrophages, as another important mechanism. LPA administration led to IL-33 augmentation in the supernatants of lung homogenates, which correlated with our findings of IL-13 in BALF in our LPA mouse model (Fig. 5d, e). At first, we hypothesized that LPA stimulated bronchial epithelial cells to produce IL-33; however, IL-33 was not detected in the media supernatants of cultured BEAS-2B cells 
stimulated by LPA (data not shown). Therefore, we focused attention on macrophages because IL-33 derived from macrophages induced IL-13 production by ILC2 and increased airway hypersensitivity in influenza-infected mice [34]. Several studies have reported that macrophages produce IL-33 $[35,36]$. In the present findings, since the number of macrophages correlated with IL-33 in the lungs, it is likely that LPA may elicit macrophagederived IL-33 production, leading to an increase in IL-13 production in the lungs in our mouse model of allergic inflammation.

Based on this, we wondered if the migration of macrophage we observed was due to a direct effect of LPA. First, we hypothesized that LPA affected macrophages directly and made them produce IL-33. However, since the level of LPA1 was higher than that of LPA2 on macrophages in BALF cells (online suppl. Table 1), we thought that LPA1 was probably more important for macrophages. A previous report also showed that LPA1 was important for macrophage infiltration, supporting our conclusion [16]. Next, in order to investigate LPA2's relationship with macrophages, we focused our attention on CCL2, which is a chemokine of monocytes, macrophages, and Tlymphocytes and is an activator of monocytes/macrophages [37]. In in vivo experiments, CCL2 production was increased in an LPA dose-dependent manner (Fig. 6d), and administration of an LPA2 antagonist affected CCL2 production in lungs with allergic inflammation (Fig. 3e). These findings suggest that LPA induced CCL2 production, leading to the infiltration of macrophages and IL-33 production. Moreover, these findings also suggest that an LPA2 antagonist suppressed the CCL2-elicited macrophage infiltration. Several previous reports showed that LPA induced the expression of CCL2 (MCP-1) in human aortic smooth muscle cells and vascular endothelial cells $[38,39]$. Based on this, our present findings suggest that the increase in CCL2 induced by LPA leads to macrophage migration and activation and increased production of IL-33. Furthermore, an LPA2 antagonist suppressed the increase of CCL2, leading us to conclude that LPA2 plays a critical role in macrophage activation in inflamed lungs.

Interestingly, although we demonstrated that the LPA/ LPA2 axis worsened allergic inflammation and that an LPA2 antagonist decreased disease, there are several reports that showed the opposite result. For example, an LPA2 agonist suppressed allergic eosinophilic bronchial inflammation [40]. Emo et al. [41] reported that LPA2 deficiency led to worse asthmatic symptoms. Thus, the effect of LPA receptor inhibition for the management of asthma is still controversial. The causes of these differences remain unknown. It is likely that the differences may be affected by the models based on the kind of antigen exposure, gene manipulation, and the timing of administration of the LPA2 antagonist. Emo et al. [41] reported that LPA2 suppressed allergic inflammation via DC. On the other hand, we demonstrated that LPA mainly acts on Th2 differentiation and macrophage infiltration, exacerbating IL-13/IL-33-mediated allergic inflammation. We could potentially explain that the difference in target cells led to the differential effect of LPA. Meanwhile, Knowlden et al. [40] reported that an LPA2 "agonist" suppressed allergic inflammation via IL-4, IL-5, and IL-10 production. In contrast, we also demonstrated that the "antagonist" suppressed IL- 5 and IL-13 production by suppressing Th2 differentiation and IL-33 production. Interestingly, both the LPA2 "agonist" and the "antagonist" suppressed the allergic inflammation via the LPA2 receptor; nevertheless, the target cells or molecular mechanisms may be different. From this point of view, we consider the LPA/LPA2 axis plays an important role in the suppression of allergic inflammation. Further examination is necessary in order to clarify the molecular mechanism.

In summary, we demonstrated that LPA was important for exacerbating allergic inflammation via $\mathrm{T}$ cell and macrophage activation in an allergic inflammation model. Moreover, LPA2 antagonist treatment decreased Th2 differentiation and production of IL-33 in the lungs, suggesting that management of LPA could be a therapeutic candidate for severe asthma. Further investigation will be required to clarify the role of LPA in airway remodeling in chronic asthma.

\section{Acknowledgements}

We thank Megumi Kume, Hitomi Umemoto, and Yuuki Morimoto (Department of Pathology and Laboratory Medicine, Institute of Medical Biosciences, Tokushima University Graduate School) for preparing histological sections and stains. We thank the Support Center for Advanced Medical Sciences, Tokushima University Graduate School of Biomedical Science, for supporting cell sorting.

\section{Statement of Ethics}

Mice were maintained in the animal facility of Tokushima University under specific pathogen-free conditions, according to the guidelines and approval of the ethics committee of our university. The approval numbers of the experimental design are T27-66 and T30-81. Their expiration dates are August 31, 2018, and September 11,2021 , respectively. 


\section{Conflict of Interest Statement}

All authors declare that they have no competing interests.

\section{Funding Sources}

All authors declare that they have no funding sources.

\section{Author Contributions}

M.K., H.O., T.T., M.A., K.K., and Y.N. contributed to conception, study design, data interpretation, and writing of the manuscript. M.K., H.O., T.T., and H.B. carried out data acquisition. H.O. carried out histopathology and IHC. K.K. carried out cell sorting and FACS analysis. M.K., H.O., and T.T. carried out analysis and interpretation. All authors read and approved the final manuscript.

\section{References}

1 Aoki J, Inoue A, Okudaira S. Two pathways for lysophosphatidic acid production. Biochim Biophys Acta. 2008;1781(9):513-8.

2 Choi JW, Herr DR, Noguchi K, Yung YC, Lee CW, Mutoh T, et al. LPA receptors: subtypes and biological actions. Annu Rev Pharmacol Toxicol. 2010;50:157-86.

3 Park GY, Lee YG, Berdyshev E, Nyenhuis S, $\mathrm{Du}$ J, Fu P, et al. Autotaxin production of lysophosphatidic acid mediates allergic asthmatic inflammation. Am J Respir Crit Care Med. 2013;188(8):928-40.

$4 \mathrm{Wu}$ C, Huang RT, Kuo CH, Kumar S, Kim CW, Lin YC, et al. Mechanosensitive PPAP2B regulates endothelial responses to atherorelevant hemodynamic forces. Circ Res. 2015; 117(4):e41-53.

5 Tager AM, LaCamera P, Shea BS, Campanella GS, Selman M, Zhao Z, et al. The lysophosphatidic acid receptor LPA1 links pulmonary fibrosis to lung injury by mediating fibroblast recruitment and vascular leak. Nat Med. 2008;14(1):45-54.

6 Montesi SB, Mathai SK, Brenner LN, Gorshkova IA, Berdyshev EV, Tager AM, et al. Docosatetraenoyl LPA is elevated in exhaled breath condensate in idiopathic pulmonary fibrosis. BMC Pulm Med. 2014;14:5.

7 Rubenfeld J, Guo J, Sookrung N, Chen R, Chaicumpa W, Casolaro V, et al. Lysophosphatidic acid enhances interleukin-13 gene expression and promoter activity in T cells. Am J Physiol Lung Cell Mol Physiol. 2006; 290(1):L66-74

8 Wang L, Knudsen E, Jin Y, Gessani S, Maghazachi AA. Lysophospholipids and chemokines activate distinct signal transduction pathways in T helper 1 and T helper 2 cells. Cell Signal. 2004;16(9):991-1000.

9 Bai Z, Cai L, Umemoto E, Takeda A, Tohya K, Komai Y, et al. Constitutive lymphocyte transmigration across the basal lamina of high endothelial venules is regulated by the autotaxin/lysophosphatidic acid axis. J Immunol. 2013;190(5):2036-48

10 Knowlden SA, Capece T, Popovic M, Chapman TJ, Rezaee F, Kim M, et al. Regulation of T cell motility in vitro and in vivo by LPA and LPA2. PLoS One. 2014;9(7):e101655.
11 Knowlden S, Georas SN. The autotaxin-LPA axis emerges as a novel regulator of lymphocyte homing and inflammation. J Immunol. 2014;192(3):851-7.

12 Saatian B, Zhao Y, He D, Georas SN, Watkins T, Spannhake EW, et al. Transcriptional regulation of lysophosphatidic acid-induced interleukin- 8 expression and secretion by $\mathrm{p} 38$ MAPK and JNK in human bronchial epithelial cells. Biochem J. 2006;393(Pt 3):657-68.

13 Toews ML, Ustinova EE, Schultz HD. Lysophosphatidic acid enhances contractility of isolated airway smooth muscle. J Appl Physiol. 1997;83(4):1216-22.

14 Georas SN, Berdyshev E, Hubbard W, Gorshkova IA, Usatyuk PV, Saatian B, et al. Lysophosphatidic acid is detectable in human bronchoalveolar lavage fluids at baseline and increased after segmental allergen challenge. Clin Exp Allergy. 2007;37(3):311-22.

15 Hashimoto T, Yamashita M, Ohata $\mathrm{H}$, Momose K. Lysophosphatidic acid enhances in vivo infiltration and activation of guinea pig eosinophils and neutrophils via a Rho/Rhoassociated protein kinase-mediated pathway. J Pharmacol Sci. 2003;91(1):8-14.

16 Swaney JS, Chapman C, Correa LD, Stebbins KJ, Bundey RA, Prodanovich PC, et al. A novel, orally active LPA(1) receptor antagonist inhibits lung fibrosis in the mouse bleomycin model. Br J Pharmacol. 2010;160(7):1699-713.

17 Huang LS, Fu P, Patel P, Harijith A, Sun T, Zhao Y, et al. Lysophosphatidic acid receptor-2 deficiency confers protection against bleomycin-induced lung injury and fibrosis in mice. Am J Respir Cell Mol Biol. 2013; 49(6):912-22.

18 Miyabe Y, Miyabe C, Iwai Y, Takayasu A, Fukuda S, Yokoyama W, et al. Necessity of lysophosphatidic acid receptor 1 for development of arthritis. Arthritis Rheum. 2013;65(8): 2037-47.

19 Smyth SS, Cheng HY, Miriyala S, Panchatcharam M, Morris AJ. Roles of lysophosphatidic acid in cardiovascular physiology and disease. Biochim Biophys Acta. 2008; 1781(9):563-70.

20 Zhao Y, Tong J, He D, Pendyala S, Evgeny B, Chun J, et al. Role of lysophosphatidic acid receptor LPA2 in the development of allergic airway inflammation in a murine model of asthma. Respir Res. 2009;10:114.
21 Takahashi K, Minami K, Otagaki S, Ishimoto K, Fukushima K, Fukushima N, et al. Lysophosphatidic acid receptor-2 (LPA2) and LPA5 regulate cellular functions during tumor progression in fibrosarcoma HT1080 cells. Biochem Biophys Res Commun. 2018; 503(4):2698-703

22 Volden PA, Skor MN, Johnson MB, Singh P, Patel FN, McClintock MK, et al. Mammary adipose tissue-derived lysophospholipids promote estrogen receptor-negative mammary epithelial cell proliferation. Cancer Prey Res. 2016;9(5):367-78.

23 Nishioka Y, Yano S, Fujiki F, Mukaida N, Matsushima K, Tsuruo T, et al. Combined therapy of multidrug-resistant human lung cancer with anti-P-glycoprotein antibody and monocyte chemoattractant protein-1 gene transduction: the possibility of immunological overcoming of multidrug resistance. Int $J$ Cancer. 1997;71(2):170-7.

24 Ogawa H, Azuma M, Muto S, Nishioka Y, Honjo A, Tezuka T, et al. IкB kinase $\beta$ inhibitor IMD-0354 suppresses airway remodelling in a Dermatophagoides pteronyssinus-sensitized mouse model of chronic asthma. Clin Exp Allergy. 2011;41(1):104-15.

25 Schmitz J, Owyang A, Oldham E, Song Y, Murphy E, McClanahan TK, et al. IL-33, an interleukin-1-like cytokine that signals via the IL-1 receptor-related protein ST2 and induces $\mathrm{T}$ helper type 2-associated cytokines. Immunity. 2005;23(5):479-90.

26 Goetzl EJ, Kong Y, Mei B. Lysophosphatidic acid and sphingosine 1-phosphate protection of $\mathrm{T}$ cells from apoptosis in association with suppression of Bax. J Immunol. 1999;162(4):2049-56.

27 Zheng Y, Kong Y, Goetzl EJ. Lysophosphatidic acid receptor-selective effects on Jurkat $\mathrm{T}$ cell migration through a Matrigel model basement membrane. J Immunol. 2001; 166(4):2317-22.

28 Zheng Y, Voice JK, Kong Y, Goetzl EJ. Altered expression and functional profile of lysophosphatidic acid receptors in mitogen-activated human blood T lymphocytes. FASEB J. 2000; 14(15):2387-9.

29 Moro K, Yamada T, Tanabe M, Takeuchi T, Ikawa $\mathrm{T}$, Kawamoto $\mathrm{H}$, et al. Innate production of $\mathrm{T}(\mathrm{H}) 2$ cytokines by adipose tissue-associated c-Kit $(+) \mathrm{Sca}-1(+)$ lymphoid cells. Nature. 2010;463(7280):540-4. 
30 Neill DR, Wong SH, Bellosi A, Flynn RJ, Daly $\mathrm{M}$, Langford TK, et al. Nuocytes represent a new innate effector leukocyte that mediates type-2 immunity. Nature. 2010;464(7293): 1367-70.

31 Anderson GP. Endotyping asthma: new insights into key pathogenic mechanisms in a complex, heterogeneous disease. Lancet. 2008;372(9643):1107-19.

32 Doherty TA, Khorram N, Lund S, Mehta AK, Croft M, Broide DH. Lung type 2 innate lymphoid cells express cysteinyl leukotriene receptor 1, which regulates TH2 cytokine production. J Allergy Clin Immunol. 2013; 132(1):205-13.

33 Xue L, Salimi M, Panse I, Mjösberg JM, McKenzie AN, Spits H, et al. Prostaglandin D2 activates group 2 innate lymphoid cells through chemoattractant receptor-homologous molecule expressed on $\mathrm{TH} 2$ cells. J Allergy Clin Immunol. 2014;133(4):1184-94.
34 Chang YJ, Kim HY, Albacker LA, Baumgarth $\mathrm{N}$, McKenzie AN, Smith DE, et al. Innate lymphoid cells mediate influenza-induced airway hyper-reactivity independently of adaptive immunity. Nat Immunol. 2011;12(7):631-8.

35 Mishra PK, Palma M, Buechel B, Moore J, Davra V, Chu N, et al. Sterile particle-induced inflammation is mediated by macrophages releasing IL-33 through a Bruton's tyrosine kinase-dependent pathway. Nat Mater. 2019; 18(3):289-97.

36 Qi F, Bai S, Wang D, Xu L, Hu H, Zeng S, et al. Macrophages produce IL-33 by activating MAPK signaling pathway during RSV infection. Mol Immunol. 2017;87:284-92.

37 Rollins BJ. Monocyte chemoattractant protein 1: a potential regulator of monocyte recruitment in inflammatory disease. Mol Med Today. 1996;2(5):198-204.
38 Hao F, Tan M, Wu DD, Xu X, Cui MZ. LPA induces IL-6 secretion from aortic smooth muscle cells via an LPA1-regulated, PKC-dependent, and p38alpha-mediated pathway. Am J Physiol Heart Circ Physiol. 2010;298(3): H974-83.

39 Takeda Y, Matoba K, Kawanami D, Nagai Y, Akamine $\mathrm{T}$, Ishizawa $\mathrm{S}$, et al. ROCK2 regulates monocyte migration and cell to cell adhesion in vascular endothelial cells. Int J Mol Sci. 2019;20(6):1331.

40 Knowlden SA, Hillman SE, Chapman TJ, Patil R, Miller DD, Tigyi G, et al. Novel Inhibitory effect of a lysophosphatidic acid 2 agonist on allergen-driven airway inflammation. Am J Respir Cell Mol Biol. 2016;54(3):402-9.

41 Emo J, Meednu N, Chapman TJ, Rezaee F, Balys M, Randall T, et al. Lpa2 is a negative regulator of both dendritic cell activation and murine models of allergic lung inflammation. J Immunol. 2012;188(8):3784-90. 